\title{
Calidad del servicio de internet y satisfacción del cliente
}

Edison Yuver Moreno Cardenas ${ }^{1}$

\begin{abstract}
RESUMEN
El objetivo principal consistió en identificar la variación del promedio de la satisfacción del cliente en proporción al promedio de la calidad del servicio de internet. La población estuvo conformada por trece clientes de la cartera corporativa del proveedor de servicios de internet América Móvil Perú S. A. C. Asimismo, se utilizaron las variables: calidad del servicio de internet y satisfacción del cliente. Se empleó el diseño no experimental de nivel correlacional, que ha sido desarrollado en la aplicación de las encuestas, las cuales brindaron resultados acerca de la variación entre las variables. La investigación ha concluido con el resultado de 0.77 , lo que indica que existe variación proporcional positiva entre ambas variables. Además, se encuentra en un nivel correlacional alto; y, al realizar el análisis del nivel de significancia bilateral de 0.000 menor a 0.05 , se concluye que el promedio de la calidad del servicio de internet varía en proporción al promedio de la satisfacción del cliente.
\end{abstract}

Palabras-claves: Calidad del servicio; internet; satisfacción del cliente; cartera corporativa.

\section{INTRODUCCIÓN}

El crecimiento de la industria de los servicios de telecomunicaciones es, tal vez, el más rápido de todos en la última década. Hay jugadores pequeños y grandes en esta industria, cada uno tratando de crear un nicho para sí mismo. No obstante, estos años hubo un avance reciente, tanto a nivel mundial como nacional, en el uso, la demanda y la implementación de los servicios de telecomunicaciones y de la tecnología de la información (TI). Aquella, junto con el uso de internet, representan oportunidades para que las empresas logren ventajas competitivas estratégicas en comparación con su competencia y puedan facilitar el movimiento de bienes y servicios de los productores a los clientes (Berisha, 2015).

Estar a la vanguardia del cambio tecnológico proporciona mejores resultados para un negocio en base a cuatro puntos positivos: apertura al mercado internacional, eficiencia de costes, facilidad y rapidez para llegar a los clientes y desarrollo de la imagen. La definición más precisa sobre el cliente la dan Gavilanez y Ortiz (2009), quienes señalan que el cliente externo es aquella persona que no pertenece a la institución, por lo tanto, se le define como toda persona natural o jurídica que requiere satisfacer una necesidad mediante la intervención de terceros. Asimismo, Domínguez (2006) señala que el cliente interno, es decir, el personal que labora en la empresa, «es receptor primario de la misión, visión y estrategias formuladas por la organización con el fin de lograr la satisfacción del cliente externo» (p. 4).

En tal sentido, la investigación analizó la correlación existente entre el promedio de la satisfacción de los clientes y el promedio de la calidad del servicio de internet en un determinado proveedor de servicio de internet (ISP). El estudio se ha limitado a trece clientes de la cartera corporativa.

La investigación tomó en cuenta que la satisfacción se produce cuando el desempeño percibido del producto coincide con las expectativas del cliente (Kotler y Keller, 2006). Por lo tanto, exis-

1 Ingeniero electrónico por la Universidad Nacional de San Antonio Abad del Cusco. Consultor independiente. Lima, Perú.

ORCID: 0000-0001-9545-4694.

E-mail: edy.morenoc@gmail.com 
te un amplio acuerdo en que la satisfacción es la sensación de placer o desilusión de una persona al comparar el desempeño percibido de un producto en relación con sus expectativas. Por ese motivo, se ha concluido que la satisfacción del cliente viene a ser el gusto que experimenta luego de haber consumido el producto o servicio entregado. Complementariamente, se describe la calidad como un equivalente de satisfacción y de superación de lo que el cliente espera del producto o servicio ofrecidos para complacer sus necesidades (Alcalde, 2009).

Un punto fundamental es la importancia de los procesos en las áreas de atención al cliente. Así, Pall (1987) define un proceso como la organización lógica de personas, materiales, energía, equipamiento e información en actividades de trabajo diseñadas con el fin de producir un resultado final requerido, ya sea producto o servicio. Por consiguiente, los procesos son piezas clave dentro de las organizaciones. Ello conlleva a que el promedio de la satisfacción esté estrechamente relacionado con las expectativas de los consumidores, los cuales ayudan a encaminar futuras investigaciones que podrían desarrollarse entre otras dimensiones con mayor precisión para mejorar las estrategias de calidad del servicio $u$ otros factores que influyan para atraer a los clientes a un mejor servicio de internet.

Finalmente, una investigación futura de naturaleza similar puede implicar un periodo y números de recopilación de datos más largos, lo cual elimina cualquier variable que pueda haber producido anomalías en este resultado.

\section{METODOLOGÍA}

La metodología en esta investigación contempla el uso de los recursos apropiados para el análisis estadístico, los cuales proporcionan una percepción más especifica del objetivo de la investigación. Para la unidad de análisis, se ha considerado al cliente corporativo; en este caso, una persona jurídica.

La presente investigación tiene un enfoque cuantitativo, tipo de estudio sustentado por Hernández, Fernández y Baptista (2010), pues la recolección de datos es equivalente a medir con diseño descriptivo; se pretende congregar información de forma independiente acerca de las variables a las que nos vamos a referir. También es de tipo correlacional, porque tiene como finalidad conocer la relación o el grado de asociación existente entre las dos variables propuestas. Además, esta investigación es no experimental, porque esta se realiza sin manipular deliberadamente las variables, es decir, se observa los fenómenos tal como se dan en su contexto natural para su análisis posterior. Como se dijo, el universo que se contempla en esta investigación ha sido conformado por trece clientes de la cartera corporativa del proveedor de servicios de internet América Móvil Perú S. A. C.

Para el análisis, se realizó un muestreo poblacional debido a que la muestra y la población fueron iguales, siendo la muestra menor a treinta individuos. De la misma manera, la investigación cumplió con las pautas de inclusión por enmarcar la población objeto de estudio, singularizando a los sujetos que entran en el estudio. Además, la información obtenida agrupa perspectivas asociadas a las actividades de recopilación, análisis y descripción de los resultados. Por ello, para la obtención de información, se elaboraron encuestas que fueron los instrumentos del estudio descriptivo, el cual precisó e identificó las interrogantes que se realizaron a la población seleccionada.

En las tablas 1 y 2, para el procesamiento de datos se muestra la operacionalización de las variables: promedio de la calidad del servicio de internet y promedio de la satisfacción del cliente.

Tabla 1. Variable promedio de la calidad del servicio de internet.

\begin{tabular}{|c|c|c|c|c|c|c|}
\hline Variable & Definición conceptual & $\begin{array}{l}\text { Definición } \\
\text { operacional }\end{array}$ & Indicadores & $\begin{array}{l}\text { Unidad de } \\
\text { medida }\end{array}$ & Escala & Valor final \\
\hline \multirow{5}{*}{$\begin{array}{l}\text { Calidad del } \\
\text { servicio de } \\
\text { Internet }\end{array}$} & \multirow{5}{*}{$\begin{array}{l}\text { La calidad del servicio de } \\
\text { Internet, es el patrón que mide } \\
\text { la efectividad y ausencia de } \\
\text { interrupción y/o afectación del } \\
\text { servicio de Internet, buscando } \\
\text { mantener los niveles de calidad } \\
\text { ofrecidos, permitiendo realizar } \\
\text { procesos continuos. }\end{array}$} & \multirow{5}{*}{$\begin{array}{l}\text { El promedio de la } \\
\text { calidad del servicio } \\
\text { de Internet referente } \\
\text { al servicio de Internet } \\
\text { percibido, en las que } \\
\text { normalmente debe } \\
\text { estar recibiendo }\end{array}$} & \multirow{5}{*}{$\begin{array}{l}\text { Nivel del } \\
\text { promedio de } \\
\text { la calidad }\end{array}$} & \multirow{5}{*}{ Likert-5 } & Muy satisfecho & \multirow{5}{*}{$\begin{array}{l}\text { Satisfecho } \\
\text { Regular } \\
\text { Insatisfecho }\end{array}$} \\
\hline & & & & & Satisfecho & \\
\hline & & & & & Medio & \\
\hline & & & & & Insatisfecho & \\
\hline & & & & & Muy Insatisfecho & \\
\hline
\end{tabular}

Fuente: Elaboración propia. 
Tabla 2. Variable promedio de la satisfacción del cliente.

\begin{tabular}{|c|c|c|c|c|c|c|}
\hline Variable & Definición conceptual & $\begin{array}{l}\text { Definición } \\
\text { operacional }\end{array}$ & Indicadores & $\begin{array}{l}\text { Unidad de } \\
\text { medida }\end{array}$ & Escala & Valor final \\
\hline \multirow{5}{*}{$\begin{array}{l}\text { Promedio de } \\
\text { la satisfacción } \\
\text { del cliente }\end{array}$} & \multirow{5}{*}{$\begin{array}{l}\text { La satisfacción del cliente cor- } \\
\text { porativo es el nivel de estado } \\
\text { de ánimo de una persona, } \\
\text { resultado de la comparación } \\
\text { del rendimiento percibido } \\
\text { del servicio de internet con } \\
\text { sus experiencias; expresa } \\
\text { que luego de la adquisición } \\
\text { del servicio de internet, los } \\
\text { clientes experimentan uno } \\
\text { de estos niveles o grados de } \\
\text { satisfacción. }\end{array}$} & \multirow{5}{*}{$\begin{array}{l}\text { El promedio de la } \\
\text { satisfacción del } \\
\text { cliente referente al } \\
\text { servicio de internet } \\
\text { percibido, en las } \\
\text { que normalmente } \\
\text { debe estar traba- } \\
\text { jando. }\end{array}$} & \multirow{5}{*}{$\begin{array}{l}\text { Nivel del } \\
\text { promedio de la } \\
\text { satisfacción }\end{array}$} & \multirow{5}{*}{ Likert-5 } & Muy satisfecho & \multirow{5}{*}{$\begin{array}{l}\text { Satisfecho } \\
\text { Regular } \\
\text { Insatisfecho }\end{array}$} \\
\hline & & & & & Satisfecho & \\
\hline & & & & & Medio & \\
\hline & & & & & Insatisfecho & \\
\hline & & & & & Muy Insatisfecho & \\
\hline
\end{tabular}

Fuente: elaboración propia.

Por otro lado, la obtención de la confiabilidad de los instrumentos fue a través del coeficiente alfa de Cronbach, el cual le otorgó el grado de confiabilidad. Asimismo, para la validez del instrumento de medición referente a cada variables, se constató la validación de cada instrumento, los cuales mostraron resultados positivos.

Según Quero (2010): «Con la creación del alfa de Cronbach, los investigadores fueron capaces de evaluar la confiabilidad de un instrumento constituido por una escala Likert» (p. 250). Cronbach y Shavelson (2004) señalaron que el coeficiente solo cubre un enfoque estrecho dentro de la problemática más amplia del análisis de confiabilidad. En la tabla 3 se ha determinado la confiabilidad de los instrumentos:

Tabla 3. Confiabilidad del cuestionario promedio de la calidad del servicio de internet.

\begin{tabular}{|c|c|}
\hline \multicolumn{2}{|c|}{ Estadísticas de fiabilidad } \\
\hline Alfa de Cronbach & $\mathrm{N}{ }^{\circ}$ de elementos \\
\hline 0,960 & 5 \\
\hline
\end{tabular}

Fuente: elaboración propia, obtenido por el programa SPSS (2018).

Se considera que el coeficiente varía entre 0 y 1 , lo cual indica que 0 representa una confiabilidad nula y 1 , confiabilidad total (Corral, 2009). Por otra parte, se tiene en cuenta que la coherencia interna es alta cuando esta se encuentra en el rango de 0.70 a 0.90 . Asimismo, los valores por debajo de 0.70 señalan una baja coherencia interna, mientras que los valores superiores a 0.90 señalan que la escala tiene varios ítems que determinan puntualmente lo mismo (Celina y Campo, 2005).

En los resultados se ha obtenido el valor $0.960 \mathrm{del}$ coeficiente alfa de Cronbach, el cual fue mayor a
0.90 , lo que muestra que es cercano a la unidad. Por lo tanto, es alto el nivel de confiabilidad del instrumento, aunque algunos ítems pudieran ser redundantes (Celina y Campo, 2005). En la siguiente tabla se determina la confiabilidad del instrumento empleado para medir la satisfacción del cliente.

Tabla 4. Confiabilidad del cuestionario promedio de la satisfacción del cliente.

\begin{tabular}{|c|c|}
\hline \multicolumn{2}{|c|}{ Estadísticas de fiabilidad } \\
\hline Alfa de Cronbach & N. ${ }^{\circ}$ de elementos \\
\hline 0,826 & 2 \\
\hline
\end{tabular}

Fuente: elaboración propia, obtenido por el programa SPSS (2018).

Se tiene que el coeficiente alfa de Cronbach ha sido superior a 0.80 , pero menor a 0.90 , lo que demuestra que el nivel de confiabilidad del instrumento es alto.

Para el análisis estadístico de las variables, se empleó el software SSPS con el fin de estimar proporciones porcentuales en tablas y figuras. De esta forma, se buscó describir la distribución de los datos, teniendo en consideración que las variables en la investigación son cualitativas ordinales. Por ello, las pruebas obtenidas que le competen son no paramétricas; y, según Giorgio (2014), «el coeficiente de correlación de Spearman es no paramétri$c o$, pues la distribución muestral no se ajusta a una distribución conocida, por lo que los estimadores muestrales no son representativos de los parámetros poblacionales» (p. 5).

De igual manera, el método estadístico consistió en saber si dos variables están correlacionadas para utilizar la correlación de Spearman (rho), puesto que las variables son ordinales. Así, los resultados de los clientes corporativos se han obtenido de las mediciones. 


\section{RESULTADOS}

Los resultados y la discusión de esta investigación se basan en factores como la afectación, interrupción, continuidad del servicio de internet y los servicios de atención al cliente corporativo. Las variables utilizadas fueron: nivel de calidad del servicio de internet y nivel de satisfacción. Los factores se han cuantificado con la ayuda de las técnicas de escalamiento de Likert de cinco puntos para una inferencia estadística efectiva. Para analizar el promedio de la satisfacción de los clientes con respecto al promedio de la calidad del servicio de internet, los encuestados han tenido que responder señalando "una cifra», según la escala de calificación. El formato de las escalas de calificación fue de 1 a 5 , que correspondía en ese mismo orden a las categorías de muy insatisfecho, insatisfecho, medio (sin opinión), satisfecho y muy satisfecho.

La figura 1 muestra el factor del promedio de la calidad del servicio de internet de los encuestados. De los trece encuestados, en un nivel bajo, el $23,8 \%$ de los clientes corporativos perciben el promedio de la calidad del servicio de internet como insatisfecho. En un nivel regular, el $23,8 \%$ de los clientes corporativos perciben el promedio de la calidad del servicio de internet como medio. En un nivel alto, el $38,46 \%$ de los clientes corporativos perciben el promedio de la calidad del servicio de internet como satisfecho.
En la siguiente tabla se verifica el coeficiente de correlación entre las variables promedio de la calidad del servicio de internet y promedio de la satisfacción del cliente; así como el nivel de significancia bilateral y la cantidad de encuestados.

Al analizar el contenido de la tabla 5, obtenido por el software SPSS, se determina un grado de correlación alta (0.77), donde se verifica que es cercano a 1 y no a 0 . Asimismo, se evidencia una correlación positiva, es decir, directa, de manera que mientras mayor sea el promedio de la calidad del servicio de internet, mayor será el promedio de la satisfacción del cliente, y viceversa. Por consiguiente, cumple lo que se propuso al elegir las variables. Al realizar el análisis del nivel de significancia bilateral, se verifica que es de 0.000 , por lo que se cumple que sea menor a $0.05(p=0.000<0.05)$, lo que significa que la correlación que se ha definido es válida. Entonces, podemos concluir en que la calidad del servicio de internet se relaciona con la satisfacción de los clientes de un proveedor de este servicio.

\section{DISCUSIÓN}

En primer lugar, el resultado de 0.77 indica que tiene un efecto de correlación positiva entre ambas variables.

En segundo lugar, el grado de correlación se halla en un nivel alto; $y$, al realizar el análisis del nivel

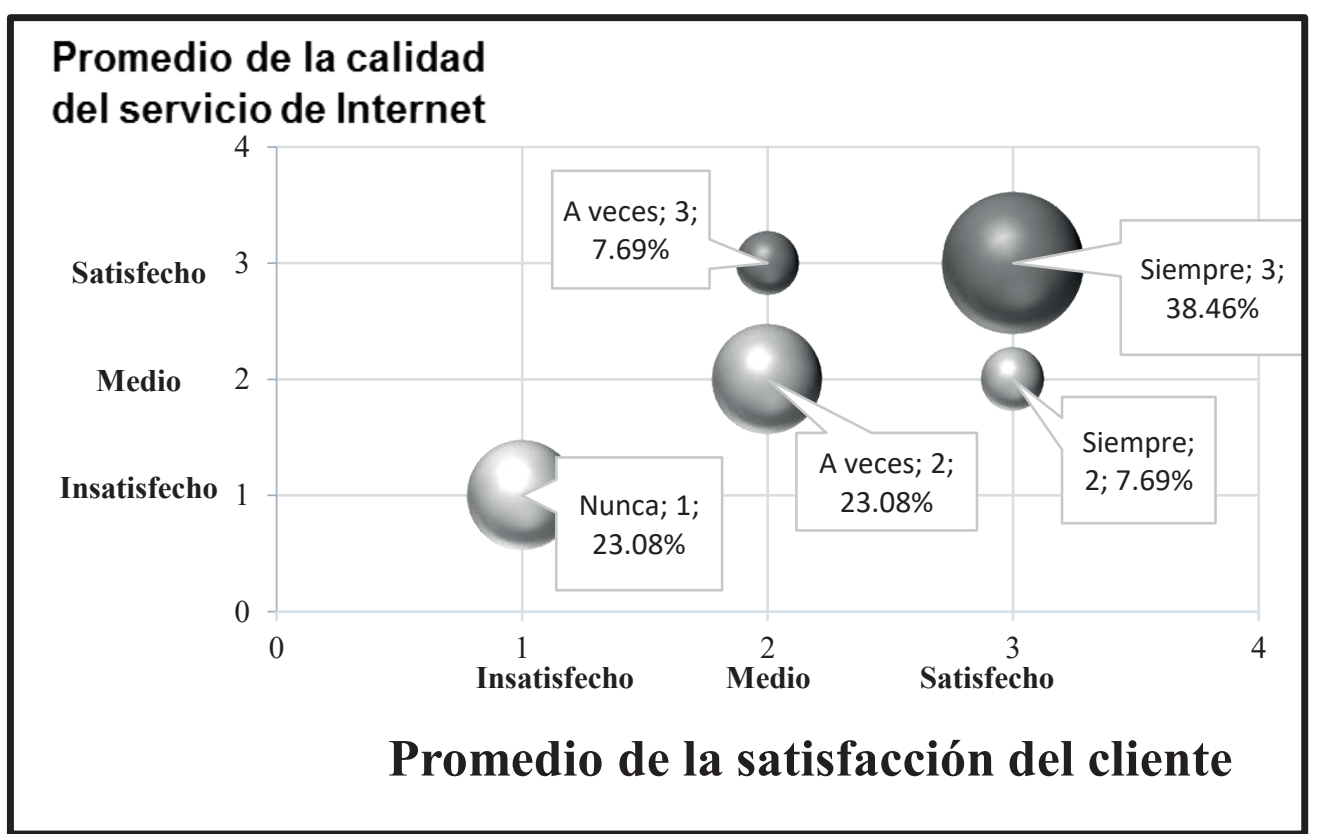

Figura 1. Diagrama de burbujas del promedio de la calidad del servicio de internet y el promedio de la satisfacción del cliente.

Fuente: elaboración propia, obtenido por el programa SPSS (2018). 
Tabla 5. Correlación del promedio de la calidad del servicio de internet y promedio de la satisfacción del cliente.

\begin{tabular}{|c|c|c|c|c|}
\hline \multicolumn{5}{|l|}{ Correlaciones } \\
\hline & & & $\begin{array}{l}\text { Promedio de la calidad del } \\
\text { servicio de internet }\end{array}$ & $\begin{array}{l}\text { Promedio de la satisfacción } \\
\text { del cliente }\end{array}$ \\
\hline \multirow{4}{*}{$\begin{array}{l}\text { Coeficiente de } \\
\text { Spearman (rho) }\end{array}$} & \multirow{2}{*}{$\begin{array}{l}\text { Promedio de la calidad } \\
\text { del servicio de internet }\end{array}$} & $\begin{array}{l}\text { Coeficiente de } \\
\text { correlación }\end{array}$ & 1000 & 0.77 \\
\hline & & Sig. (bilateral) & * & 0.001 \\
\hline & \multirow{2}{*}{$\begin{array}{l}\text { Promedio de la satisfacción } \\
\text { del cliente }\end{array}$} & $\begin{array}{l}\text { Coeficiente de } \\
\text { correlación }\end{array}$ & 0.77 & 1000 \\
\hline & & Sig. (bilateral) & 0.001 & * \\
\hline
\end{tabular}

Fuente: elaboración propia, obtenido por el programa SPSS (2018).

* La correlación es significativa en el nivel 0.01 ( 2 colas), $n=13$ empresas.

de significancia bilateral de 0.000 menor a 0.05 , se concluye en que la calidad del servicio de internet influye en la satisfacción de los clientes del proveedor de servicios de internet América Móvil Perú S. A. C. Esto demuestra que del promedio de la satisfacción de los clientes corporativos, en un nivel bajo, el $23.8 \%$ de estos perciben al promedio de la calidad del servicio de internet como insatisfecho. En un nivel regular, el $23.8 \%$ de los clientes corporativos perciben el promedio de la calidad del servicio de internet como medio. En un nivel alto, el $38.46 \%$ de los clientes corporativos perciben el promedio de la calidad del servicio de internet como satisfecho.

El presente estudio demuestra lo expuesto por Sumathisri, Muthumeenakshi y Anand (2014), pues concuerda al expresar que existe una correlación entre la satisfacción del cliente y los factores del servicio de internet, como la continuidad de este servicio y la atención al cliente. Asimismo, para lograr una satisfacción óptima del cliente, es imprescindible reducir los tiempos de afectación en el servicio de internet preferentemente sin interrupciones al mes, las cuales engloba una gestión que se aplica al enlace de internet. El principal objetivo de esa gestión es perfeccionar la satisfacción, optimizar los tiempos de operación, ausentar las interrupciones del servicio de internet y garantizar un flujo constante de este servicio hacia los clientes. Para lograr este objetivo, es necesario conocer el proceso más crítico y definirlo de forma correspondiente.

La presente investigación reafirma lo propuesto por Montes (2017), ya que concuerda en la importancia del costo del servicio, y por ser un sector corporativo tiene un mayor costo del servicio de internet. Además de ello, la atención es más exclusiva y rápida ante una interrupción o afectación de este servicio frente a otros sectores dentro de sus diferentes carteras de clientes. Esto se ve reflejado en los tiempos de restablecimiento del servicio de internet por parte del proveedor.

\section{CONCLUSIONES}

1. Esta investigación exploró el promedio de la satisfacción de los clientes hacia el proveedor de servicios de internet seleccionado: América Móvil Perú S. A. C. El estudio contempló trece clientes de la cartera corporativa. A través de esta investigación se buscó conocer la satisfacción de los clientes y la calidad del servicio de internet. Asimismo, el análisis de los datos de la investigación mostró que el promedio de la calidad del servicio de internet es un factor predictivo importante para el promedio de la satisfacción del cliente, el cual tiene un efecto de correlación positivo y significativo.

2. Las investigaciones adicionales podrían desarrollar las otras dimensiones de manera más precisa para mejorar la calidad del servicio de internet $u$ otros factores que influyan para atraer clientes corporativos para el servicio de internet. Por lo tanto, una investigación futura de naturaleza similar puede conllevar un periodo de recolección de datos y números más grandes, lo que luego podría eliminar cualquier variable que haya producido anomalías en este resultado.

\section{RECOMENDACIONES}

- Se recomienda promover el ingenio para alcanzar objetivos específicos de innovación y generar mejoras en el servicio de internet que aumente la satisfacción de los clientes. Además, se debe analizar que, en la actualidad, el ingenio es un factor fundamental para la subsistencia de cualquier compañía, el cual facilita resguardar un nivel competitivo en el mercado globalizado haciendo frente a la evolución del mismo. 
- Se recomienda desarrollar una mejor estrategia de atención al cliente, con la finalidad de actuar rápido ante reclamos del servicio de internet de los clientes. Se analiza la situación actual, y se encuentra que la competencia en el sector corporativo ha aumentado en los últimos años; es decir, han surgido nuevos proveedores del servicio de internet, los cuales han reducido la tasa de mercado corporativo frente a los cambios que estos han ido desarrollando.

- Se recomienda realizar otra investigación en sectores diferentes al sector corporativo, a fin de obtener un bosquejo mayor de la satisfacción de los clientes en los diferentes sectores, con el propósito de desarrollar objetivos estratégicos para cada sector.

- Fomentar el desarrollo de una investigación sobre la influencia de la competencia en el sector corporativo en los servicios de internet corporativo, analizando sus objetivos y tasas de mercado actual, con la finalidad de precisar la situación real y conocer qué horizontes va tomando.

\section{AGRADECIMIENTOS}

A la Universidad Nacional Mayor de San Marcos, por permitirnos realizar esta investigación que tuvo como fin presentar un aporte en el área de la producción y gestión.

\section{REFERENCIAS BIBLIOGRÁFICAS}

[1] Alcalde, P. (2009). Calidad. Madrid, España: Paraninfo.

[2] Berisha-Shaqiri, A. (2015). Impact of information technology and internet in businesses. Academic Journal of Business, Administration, Law and Social Sciences, 1(1), 73-79. Recuperado de https://www.researchgate.net/profile/Aferdita Berisha-Shaqiri/publication/287205733_ Impact_of_Information_Technology_ and_Internet_in_Businesses I links/56731c9d08aē04d9̄b099c1ad/Impactof-Information-Techno logy-and-Internet-inBusinesses.pdf.

[3] Boccardo, G. y Lizama, P. (2014). Guía de asociación entre variables (Pearsony Spearman en SPSS). Recuperado de https://es.scribd. com/document/293979001/Coeficientes-deAsociaci-n-Pearson-y-Spearman-en-SPSS.

[4] Celina, H. y Campo, A. (2005). Aproximación al uso del coeficiente alfa de Cronbach. Revista Colombiana de Psiquiatria, 34(4), 572-580.
Recuperado de https://www.redalyc.org/ pdf/806/80634409.pdf.

[5] Corral, Y. (2009). Validez y confiabilidad de los instrumentos de investigación para la recoleccion de datos. Revista Ciencias de la Educacion, 19(33), 228-247. Recuperado de http://servicio.bc.uc.edu.ve/educacion/revista/ n33/art12.pdf.

[6] Cronbach, L. y Shavelson, R. (2004). My current thoughts on coefficient alpha and successor procedures. Educational and Psychological Measurement, 64(3), 391-418. Recuperado de https://journals.sagepub.com/ doi/10.1177/0013164404266386.

[7] Domínguez, C. (2006). El servicio invisible: fundamento de un buen servicio al cliente. Bogotá, Colombia: Ecoe.

[8] Gavilanez, F. y Ortiz, S. (2009). Estudio de satisfacción en la calidad del servicio para medir la relación que existe entre el cliente interno y el cliente externo de la dirección provincial del Guayas del instituto ecuatoriano de seguridad social (IESS). (Tesis de licenciatura). Escuela Superior Politécnica del Litoral, Guayaquil.

[9] Hernández, R.; Fernández, C. y Baptista, P. (2010). Metodología de la investigación. México, D. F., México: McGraw-Hill.

[10] Kotler, P. y Keller, K. (2006). Dirección de mercadotecnia. México, D. F., México: Pearson Educación.

[11] Montes, H. (2017). Nivel de satisfacción de los clientes de telefonía celular de Claro en la ciudad de lquitos, periodo diciembre 2016. (Tesis de maestría). Universidad Nacional de la Amazonía Peruana, Iquitos. Recuperado de http://repositorio.unapiquitos.edu.pe/ bitstream/handle/UNAP/5000/Harry_Tesis_ Maestría_2017.pdf?sequence=4\&isAllowed=y.

[12] Pall, G. (1987). Quality process management. Nueva Jersey, EE. UU.: Prentice-Hall.

[13] Quero, M. (2010). Confiabilidad y coeficiente Alpha de Cronbach. Telos. Revista de Estudios Interdisciplinarios en Ciencias Sociales, 12(2), 248-252. Recuperado de https://www.redalyc. org/pdf/993/99315569010.pdf.

[14] Sumathisri, B., Muthumeenakshi, M. yAnand, S. (2014). An analysis on customers' satisfaction towards internet service providers. European Journal of Business and Social Sciences, 3(3), 68-81. 


\section{Internet service quality and customer satisfaction}

Edison Yuver Moreno Cardenas ${ }^{1}$

\section{ABSTRACT}

The main objective was to identify the variation of the average of customer satisfaction in proportion to the average of Internet service quality. The population consisted of 13 corporate portfolio customers of Internet service provider América Móvil Perú S. A. C. Likewise, the variables used were quality of Internet service and customer satisfaction. The non-experimental correlational design was used for this purpose in the form of surveys, which provided results regarding the variation between the variables. The research obtained a result of 0.77 , which indicates that there is a positive proportional variation between both variables. Furthermore, there is a high level of correlation and, when performing the analysis, the bilateral significance level was 0.000 , less than 0.05 . It is concluded that the average of Internet service quality varies in proportion to the average of customer satisfaction.

Keywords: Service quality; Internet; customer satisfaction; corporate portfolio.

\section{INTRODUCTION}

The telecommunications services industry has been perhaps the fastest-growing sector in the last decade. There are small and large players in this industry, each striving to create a niche for themselves. However, recently, an increase regarding the use, demand, and implementation of telecommunications services and information technology (IT) was observed, both at global and national level. Information technology together with the use of the Internet represent opportunities for companies to achieve strategic competitive advantages compared to their competitors, and facilitate the movement of goods and services from producers to customers (Berisha, 2015).

For a business, being at the forefront of technological change offers better results based on four points: opening to the international market, cost-efficiency, ease and speed to reach customers and improvement of the company's image. Gavilanez and Ortiz (2009) provide the most precise definition of customer; they state that the external customer is a person who does not pertain to the institution, therefore, is defined as any individual or legal entity that needs to satisfy a need through the intervention of a third party. Similarly, Domínguez (2006) indicates that the internal customer, namely personnel working at the company, "es receptor primario de la misión, visión y estrategias formuladas por la organización con el fin de lograr la satisfacción del cliente externo" [is the primary recipient of the mission, vision and strategies devised by the company in order to achieve external customer satisfaction] (p. 4).

In this regard, the study analyzed the correlation between the average of customer satisfaction and Internet service quality of a particular Internet service provider (ISP). The study is limited to thirteen corporate portfolio customers. This study took into account the fact that satisfaction occurs when the perceived performance of the product matches the expectations of the customers (Kotler \& Keller, 2006). Thus, there is a broad consensus that sa-

1 Electronic Engineer from Universidad Nacional de San Antonio Abad del Cusco. Independent consulant. Lima, Perú.

ORCID: 0000-0001-9545-4694.

E-mail: edy.morenoc@gmail.com 
tisfaction is the sensation of pleasure or disappointment that people get when comparing the perceived performance of a product with their expectations. For this reason, it was concluded that customer satisfaction is the joy experienced after having consumed a product or used a service. In addition, quality is described as an equivalent of satisfaction and exceedance of customers' expectations regarding the product or service offered to meet their needs (Alcalde, 2009).

The importance of the processes within the customer service areas is a fundamental point. Pall (1987) defines a process as the logical organization of people, materials, energy, equipment, and information into work activities designed to produce a required end result, be it a product or service. Therefore, customer service processes are key elements within organizations. This implies that the satisfaction average is closely related to customers' expectations, which helps to direct future research that may address other dimensions with greater precision to improve service quality strategies or other factors that contribute to attracting customers to the Internet service.

Finally, future research of similar nature may involve a longer period of time and the collection of more data, which eliminates any variable that may have produced anomalies in this result.

\section{METHODOLOGY}

The methodology of this study considers the use of appropriate resources for statistical analysis, which provides a more specific perception of the research objective. The analysis unit was the corporate customer, a legal entity in this case.

This study is quantitatively-focused, as explained by Hernández, Fernández and Baptista (2010), because data is collected; descriptive, because it intends to collect data independently regarding each variable; correlational, because it aims to determine the relationship or degree of association between the two proposed variables; and non-experimental, because the variables are not deliberately manipulated, thus, phenomena are observed as they occur in their natural context for further analysis. As mentioned above, the research population consists of 13 corporate portfolio customers of the Internet service provider América Móvil Perú S. A. C.

For the analysis, a population sampling was conducted, as both the sampling and population were equal, with the sample being comprised of less than thirty individuals. Similarly, the research complied with the inclusion conditions singling out the subjects considered in the study. In addition, the obtained information groups perspectives related to the gathering, analysis and description of results. Therefore, surveys were the instruments used in this descriptive study to gather data, where all questions addressed to the selected population were outlined.

Table 1 and 2 show the operationalization of the variables "average of Internet service quality" and "average of customer satisfaction" for data processing.

On the other hand, Cronbach's alpha was used to estimate the reliability of the instruments, which was confirmed. Additionally, all instruments used to measure each variable were validated.

According to Quero (2010), "Con la creación del alfa de Cronbach, los investigadores fueron capaces de evaluar la confiabilidad de un instrumento constituido por una escala Likert" [with the formulation of Cronbach's alpha, researchers were able to assess the reliability of a Likert-scale instrument] (p.50). Cronbach and Shavelson (2004) stressed that the coefficient only covers a narrow approach within the

Table 1. Variable average of Internet service quality.

\begin{tabular}{|c|c|c|c|c|c|c|}
\hline Variable & Conceptual definition & Operational definition & Indicators & $\begin{array}{l}\text { Unit of } \\
\text { measure }\end{array}$ & Score & Final score \\
\hline \multirow{5}{*}{$\begin{array}{l}\text { Internet } \\
\text { service } \\
\text { quality }\end{array}$} & \multirow{5}{*}{$\begin{array}{l}\text { Internet service quality is the } \\
\text { parameter that measures effec- } \\
\text { tiveness and absence of service } \\
\text { interruptions and/or problems, } \\
\text { in order to maintain the quality } \\
\text { levels offered, allowing to carry } \\
\text { out continuous processes. }\end{array}$} & \multirow{5}{*}{$\begin{array}{l}\text { Average of Internet ser- } \\
\text { vice quality compared } \\
\text { to perceived Internet } \\
\text { service received under } \\
\text { normal conditions. }\end{array}$} & \multirow{5}{*}{$\begin{array}{l}\text { Level of } \\
\text { quality } \\
\text { average }\end{array}$} & \multirow{5}{*}{ Likert-5 } & Very satisfied & \multirow{5}{*}{$\begin{array}{l}\text { Satisfied } \\
\text { Regular } \\
\text { Unsatisfied }\end{array}$} \\
\hline & & & & & Satisfied & \\
\hline & & & & & Neutral & \\
\hline & & & & & Unsatisfied & \\
\hline & & & & & Very unsatisfied & \\
\hline
\end{tabular}

Source: Prepared by the authors. 
Table 2. Variable average of customer satisfaction.

\begin{tabular}{|c|c|c|c|c|c|c|}
\hline Variable & Conceptual definition & $\begin{array}{c}\text { Operational } \\
\text { definition }\end{array}$ & Indicators & $\begin{array}{c}\text { Unit of } \\
\text { measure }\end{array}$ & Score & Final score \\
\hline \multirow{5}{*}{$\begin{array}{l}\text { Average of } \\
\text { customer } \\
\text { satisfaction }\end{array}$} & \multirow{5}{*}{$\begin{array}{l}\text { Corporate customer satisfac- } \\
\text { tion is the level of the state } \\
\text { of mind of a person, which } \\
\text { results from the perceived } \\
\text { Internet service performance } \\
\text { compared to his/her experien- } \\
\text { ces. It expresses that after } \\
\text { acquiring the Internet service, } \\
\text { customer experience one of } \\
\text { these levels or degrees of } \\
\text { satisfaction. }\end{array}$} & \multirow{5}{*}{$\begin{array}{l}\text { The average of cus- } \\
\text { tomer satisfaction } \\
\text { compared to the } \\
\text { perceived Internet } \\
\text { service, under } \\
\text { normal operating } \\
\text { conditions. }\end{array}$} & \multirow{5}{*}{$\begin{array}{l}\text { Level of satis- } \\
\text { faction average }\end{array}$} & \multirow{5}{*}{ Likert-5 } & Very satisfied & \multirow{5}{*}{$\begin{array}{l}\text { Satisfied } \\
\text { Regular } \\
\text { Unsatisfied }\end{array}$} \\
\hline & & & & & Satisfied & \\
\hline & & & & & Neutral & \\
\hline & & & & & Unsatisfied & \\
\hline & & & & & Very unsatisfied & \\
\hline
\end{tabular}

Source: Prepared by the authors.

broader problem of the reliability analysis. Table 3 shows the reliability of the instruments used.

Table 3. Reliability of the questionnaire average of Internet service quality.

\begin{tabular}{|c|c|}
\hline \multicolumn{2}{|c|}{ Reliability Statistics } \\
\hline Cronbach's alpha & No. of items \\
\hline 0.960 & 5 \\
\hline
\end{tabular}

Source: Prepared by the authors using SPSS software (2018).

Coefficient ranges from 0 and 1 , with 0 indicating no reliability and 1 total reliability (Corral, 2009). Additionally, there is high internal consistency when coefficient ranges between 0.70 and 0.90 . Also, values below 0.70 indicate low internal consistency, whereas values higher than 0.90 indicate that several items of the scale determine the same (Celina \& Campos, 2005).

A Cronbach's alpha coefficient of 0.960 was obtained, which is higher than 0.90 , indicating it is close to 1 . Accordingly, the instrument has a high degree of reliability, although some items may be redundant (Celina \& Campos, 2005). Reliability of the instrument used to measure customer satisfaction is shown in the following table.

Table 4. Reliability of the questionnaire average of customer satisfaction.

\begin{tabular}{|c|c|}
\hline \multicolumn{2}{|c|}{ Reliability Statistics } \\
\hline Cronbach's alpha & No. of items \\
\hline 0.826 & 2 \\
\hline
\end{tabular}

Source: Prepared by the authors using SPSS software (2018).

Cronbach's alpha coefficient is higher than 0.80 , but less than 0.90 , which shows that the reliability of the instrument is high.
For the analysis of variables, statistical software SPSS was used in tables and figures to estimate percentage proportions. In this way, it was possible to describe data distribution bearing in mind that the variables analyzed are ordinal and qualitative. For this reason, the tests obtained are non-parametric, and according to Giorgio (2014), "el coeficiente de correlación de Spearman es no paramétrico, pues la distribución muestral no se ajusta a una distribución conocida, por lo que los estimadores muestrales no son representativos de los parámetros poblacionales" [Spearman's correlation coefficient is non-parametric, as sampling distribution does not correspond to a known distribution, thus sample estimators do not represent population parameters] (p. 5).

Similarly, the statistical method was to determine if both variables were correlated to use Spearman's rho correlation, as both variables are ordinal. In this way, results regarding corporate customers were obtained from the measurements.

\section{RESULTS}

The results and discussion of this research are based on factors such as Internet service incidents, interruption, flow and corporate customer services. The variables considered were level of Internet service quality and level of customer satisfaction. Factors were quantified through 5-point Likert scale techniques for an effective statistical inference. To analyze the customer satisfaction average with respect to Internet service quality, respondents had to provide "a figure", in accordance with the rating scale. A rating scale of 1 to 5 was used, and categories were in that same order: very unsatisfied, unsatisfied, neutral (no opinion), satisfied and very satisfied. 
Figure 1 shows the factor of the respondents regarding Internet service quality average. Of thirteen respondents, at a low level, $23.8 \%$ of corporate customers perceive the Internet service quality average as unsatisfactory; at a regular level, $23.8 \%$ perceive the Internet service quality average as standard; at a high level, $38.46 \%$ of corporate customers perceive the Internet service quality average as satisfactory.

The correlation coefficient between variables average of Internet service quality and average of customer satisfaction, as well as the two-tailed significance level, and the number of respondents are shown in the following table.

After analyzing the contents of Table 5, obtained using SPSS software, a high degree of correlation equal to 0.77 is determined, thus, confirming the value is close to 1 and not to 0 . A positive, direct correlation is also evidenced, so that the higher the average of Internet service quality, the higher the average of customer service, and viceversa. Therefore, the selection of variables was justified. A significance value of 0.000 is found upon conducting the two-tailed significance test, confirming it is less than $0.05 \quad(p=0.000<0.05)$, which indicates that the correlation is valid. It can be concluded that Internet quality service is related to customer satisfaction.

\section{DISCUSSION}

First, the result 0.77 indicates that positive correlation exists between both variables.

Second, correlation is at a high level. Upon conducting the two-tailed significance test, a significance value of 0.000 , less than 0.05 , was obtained; thus concluding that Internet service quality has an impact on the satisfaction of customers of Internet service provider América Móvil Perú S. A. C. This shows that, at a low level, $23.8 \%$ of corporate customers perceive the Internet service quality average as unsatisfactory; at a regular level, $23.8 \%$ perceive the Internet service quality average as standard; at a high level, $38.46 \%$ of corporate customers perceive the Internet service quality average as satisfactory.

This study demonstrates the statements made by Sumathisri, Muthumeenakshi and Anand (2014), as it concurs that there is correlation between customer satisfaction and factors of Internet service, such as service continuity and customer service. In addition, in order to achieve the highest levels of customer satisfaction, it is imperative to reduce the amount of internet service interruptions and issues, which includes the management of the Internet connection. The main objectives of management are to

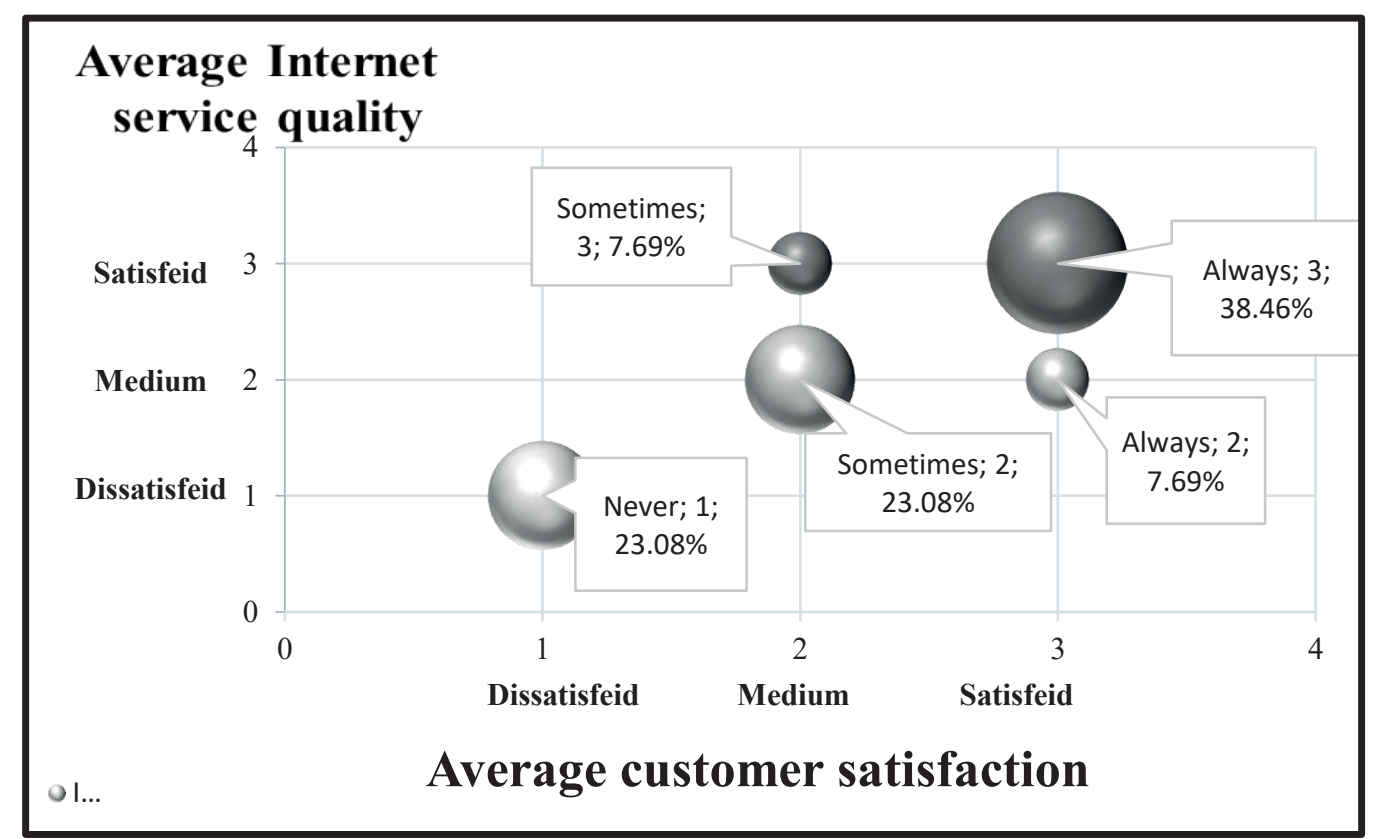

Figure 1. Bubble diagram depicting the average of Internet service quality and customer satisfaction average.

Source: Prepared by the authors using SPSS software (2018). 
Tabla 5. Correlation between the average of Internet service quality and average of customer satisfaction.

\begin{tabular}{|c|c|c|c|c|}
\hline \multicolumn{5}{|c|}{ Correlations } \\
\hline & & & $\begin{array}{l}\text { Average of Internet } \\
\text { service quality }\end{array}$ & $\begin{array}{l}\text { Average of customer } \\
\text { satisfaction }\end{array}$ \\
\hline \multirow{4}{*}{ Spearman's rho } & \multirow{2}{*}{$\begin{array}{l}\text { Average of Internet service } \\
\text { quality }\end{array}$} & Correlation coefficient & 1000 & 0.77 \\
\hline & & Sig. (2-tailed) & * & 0.001 \\
\hline & \multirow{2}{*}{ Average of customer satisfaction } & Correlation coefficient & 0.77 & 1000 \\
\hline & & Sig. (2-tailed) & 0.001 & * \\
\hline
\end{tabular}

Source: Prepared by the authors using SPSS software (2018).

${ }^{*}$ Correlation is significant at the 0.01 level (2-tailed), $n=13$ companies.

enhance satisfaction, minimize operation times, eliminate Internet service interruptions, and guarantee a steady service to customers. It is necessary to be familiarized with the most critical process and define it accordingly in order to achieve said objectives.

This study ratifies the statement made by Montes (2017), as it concurs that service cost is important, which is higher as it is provided to a corporate sector; as a result, assistance is faster and more exclusive in case of service interruption or issues compared to that provided to customers of other sectors included in the provider's customer portfolio. This is reflected in service restoration times.

\section{CONCLUSIONS}

1. This research studied the customer satisfaction average of the Internet service provider América Móvil Perú S. A. C. Thirteen corporate portfolio customers were considered in this analysis. The objective of this research was to determine customer satisfaction and Internet service quality. The analysis of research data showed that the average of Internet service quality is an important predictive factor for the average of customer satisfaction, which has a positive and significant correlation effect.

2. Other research studies could address other dimensions with greater precision to improve service quality or other factors that contribute to attract corporate customers to the Internet service. Therefore, future research of similar nature may involve a longer period of time and the collection of more data, which would eliminate any variable that may have produced anomalies in this result.

\section{RECOMMENDATIONS}

- Promote inventiveness to achieve specific innovation objectives and improve Internet service to increase customer satisfaction. Also, it should be noted that inventiveness is a fundamental factor for the survival of any company at present; it helps to maintain a competitive level in a constantly-evolving, globalized market.

- Devise a better customer service strategy in order to respond quickly to customer complaints regarding Internet service. After analyzing the current situation, it was determined that competition in the corporate sector has increased in recent years; thus, there are new Internet service providers, who have reduced the corporate market rate due to the changes they have developed.

- Conduct research in areas different from the corporate sector to obtain a larger outline regarding customer satisfaction and devise strategic objectives for each sector.

- Promote research concerning the impact of competition in the corporate sector on corporate Internet service, analyzing objectives and current market rates in order to define the current situation and discover the ways in which it is evolving.

\section{ACKNOWLEDEGMENTS}

To the Universidad Nacional Mayor de San Marcos, for allowing us to conduct this research whose purpose was to make a contribution to the production and management sector. 


\section{REFERENCES}

[1] Alcalde, P. (2009). Calidad. Madrid, Spain: Paraninfo.

[2] Berisha-Shaqiri, A. (2015). Impact of information technology and internet in businesses. Academic Journal of Business, Administration, Law and Social Sciences, 1(1), 73-79. Retrieved from https://www.researchgate.net/profile/Aferdita Berisha-Shaqiri/publication/287205733 Impact_of_Information_Technology_ and_Internet_in_Businesses I links/56731c9d08ae04d9b099c1ad/Impactof-Information-Techno logy-and-Internet-inBusinesses.pdf.

[3] Boccardo, G. \& Lizama, P. (2014). Guía de asociación entre variables (Pearson y Spearman en SPSS). Retrieved from https://es.scribd. com/document/293979001/Coeficientes-deAsociaci-n-Pearson-y-Spearman-en-SPSS.

[4] Celina, H. \& Campo, A. (2005). Aproximación al uso del coeficiente alfa de Cronbach. Revista Colombiana de Psiquiatria, 34(4), 572580. Retrieved from https://www.redalyc.org/ pdf/806/80634409.pdf.

[5] Corral, Y. (2009). Validez y confiabilidad de los instrumentos de investigación para la recoleccion de datos. Revista Ciencias de la Educacion, 19(33), 228-247. Retrieved from http://servicio.bc.uc.edu.ve/educacion/revista/ n33/art12.pdf.

[6] Cronbach, L. \& Shavelson, R. (2004). My current thoughts on coefficient alpha and successor procedures. Educational and Psychological Measurement, 64(3), 391-418. Retrieved from https://journals.sagepub.com/ doi/10.1177/0013164404266386.
[7] Domínguez, C. (2006). El servicio invisible: fundamento de un buen servicio al cliente. Bogotá, Colombia: Ecoe.

[8] Gavilanez, F. \& Ortiz, S. (2009). Estudio de satisfacción en la calidad del servicio para medir la relación que existe entre el cliente interno y el cliente externo de la dirección provincial del Guayas del instituto ecuatoriano de seguridad social (IESS). (Undergraduate thesis). Escuela Superior Politécnica del Litoral, Guayaquil.

[9] Hernández, R.; Fernández, C. \& Baptista, P. (2010). Metodología de la investigación. Mexico, D. F., Mexico: McGraw-Hill.

[10] Kotler, P. \& Keller, K. (2006). Dirección de mercadotecnia. Mexico, D. F., Mexico: Pearson Educación.

[11] Montes, H. (2017). Nivel de satisfacción de los clientes de telefonía celular de Claro en la ciudad de lquitos, periodo diciembre 2016. (Master's thesis). Universidad Nacional de la Amazonía Peruana, Iquitos. Retrieved from http://repositorio.unapiquitos.edu.pe/ bitstream/handle/UNAP/5000/Harry_Tesis_ Maestría_2017. pdf?sequence=4\&isAllowed=y.

[12] Pall, G. (1987). Quality process management. New Jersey, U.S.A.: Prentice-Hall.

[13] Quero, M. (2010). Confiabilidad y coeficiente Alpha de Cronbach. Telos. Revista de Estudios Interdisciplinarios en Ciencias Sociales, 12(2), 248-252. Retrieved from https://www.redalyc. org/pdf/993/99315569010.pdf.

[14] Sumathisri, B., Muthumeenakshi, M. \& Anand, S. (2014). An analysis on customers' satisfaction towards internet service providers. European Journal of Business and Social Sciences, 3(3), 68-81. 